\title{
Signals from the Dutch national spontaneous reporting system: Characteristics and regulatory actions
}

\author{
Florence van Hunsel ${ }^{1}$ @ | Emma de Jong ${ }^{1,2}$ | Liana Gross-Martirosyan ${ }^{3}$ | \\ Jarno Hoekman ${ }^{2}$
}

${ }^{1}$ Netherlands Pharmacovigilance Centre Lareb, 's Hertogenbosch, The Netherlands

${ }^{2}$ Utrecht University, Utrecht, The Netherlands

${ }^{3}$ Medicines Evaluation Board, Utrecht, The Netherlands

\section{Correspondence}

Florence van Hunsel, Netherlands Pharmacovigilance Centre Lareb, Goudsbloemvallei 7, 3552MH's

Hertogenbosch, the Netherlands +T 031736469700 .

Email: f.vanhunsel@lareb.nl

\begin{abstract}
Purpose: The aim of the study is to characterise safety signals based on the Dutch spontaneous reporting system (SRS) and to investigate the association between signal characteristics and Product Information (PI) update stratified by approval type: centrally authorised products (CAPs) versus nationally and decentralised authorised products (NAPs).
\end{abstract}

Methods: This study evaluates the full cohort of signals disseminated from the Dutch SRS in the period from 2008 to 2017. Each retrieved signal was characterised on a number of aspects. The signal management process from signal generation to a potential PI update was analysed in four steps: (1) signal characterisation; (2) proposed actions by the Dutch national competent authority (NCA) for the signals; (3) presence of PI update (yes/no) and association with signal characteristics; (4) timing from the moment the signal was issued to PI update. For step 1-3 we stratified products in CAPs and NAPs.

Results: Of all signals, $88.7 \%$ led to a proposed regulatory action by the NCA. Signals from the Dutch SRS for CAPs versus NAPs more often concerned biologicals, important medical events, class effects and shorter periods since marketing authorization. We detected PI updates for $26.2 \%$ of CAP signals and $61.3 \%$ of NAP signals.

Conclusions: The Dutch SRSs remains an important source of signals. There are some notable differences in the characteristics of signals for CAPs versus NAPs. Signals for NAPs more frequently led to PI updates.

\section{Why is this important?}

While previous studies showed that the characteristics of drug safety signals from a variety of sources may affect regulatory actions, there is little information on how characteristics of safety signals derived specifically from national spontaneous reporting systems are associated with regulatory actions. The aim of the study is to characterise signals based on the Dutch spontaneous reporting system and to investigate the association between signal characteristics and Product Information updates for products with a centralised marketing authorization and those without. 


\section{KEYWORDS}

drug safety signals, marketing authorization, national competent authorities,

pharmacoepidemiology, pharmacovigilance, spontaneous reporting system

\section{INTRODUCTION}

Spontaneous reports of adverse events (AEs) are a cornerstone of pharmacovigilance and one of the main methods to detect new safety signals once drugs are authorised on the market. ${ }^{1-4}$ A safety signal comprises of information on a new or known adverse event that is potentially caused by a medicine and that warrants further investigation. Signals can be generated from several sources such as spontaneous reports, clinical studies and the scientific literature. ${ }^{5}$

In the European Union, National Competent Authorities (NCAs) maintain their own spontaneous reporting system (SRS) to facilitate $A E$ reporting and the generation of drug safety signals at national level. Spontaneous reports of all Member States are also transmitted to Eudravigilance which is a centralised database of spontaneous reports maintained by the European Medicines Agency (EMA) on behalf of the European Union pharmacovigilance network. ${ }^{6}$ The EMA is in the lead for signal detection of centrally authorised products (CAPs), with the Pharmacovigilance Risk Assessment Committee (PRAC) involved in further management steps such as validation assessment and decision making. The NCAs lead signal detection for substances authorised via the decentralised procedure or mutual recognition procedure (here referred to as nationally authorised products (NAPs). ${ }^{6}$

In the Netherlands, the Medicines Evaluation Board (MEB) is the NCA responsible for drug safety signal management. The spontaneous reporting system for AEs, however, is maintained by the Netherlands Pharmacovigilance Centre Lareb, a separate organisation working in close collaboration with the MEB. All spontaneous reports are reviewed by Lareb and those with potential signal value undergo detailed analysis. In these analyses the clinical quality of the cases is reviewed, together with a study of the existing literature and possible mechanism. Safety signals are then disseminated to MEB who can take autonomous regulatory actions or forward the signal for further evaluation to the PRAC or lead member states (LMS) of NAPs when the NAP is authorised in more than one Member State. ${ }^{7}$

A previous study investigating 125 drug safety signals concerning 96 medicinal products discussed at PRAC between July 2012 and December 2013 found that $62 \%$ were triggered by spontaneous reports. Of all signals, $48 \%$ were identified by national member states and $43 \%$ originated from substances contained only in NAPs. ${ }^{2}$ Another study investigating signals discussed at PRAC between 2012 and 2016 found that the presence of evidence in multiple types of data sources; mechanistic plausibility of the drug-event association; seriousness of the event; and age of drugs $\leq 5$ years were associated with the decision to change the Product information (PI), including both the Summary of Product Characteristics and Patient Information Leaflet. $^{4}$

\section{Key Points}

- National Spontaneous Reporting Systems (SRS) and National Competent Authorities in the European Union have an important role in detecting safety signals, especially for products without a centralised marketing authorization.

- Of all safety signals from the Dutch SRS, 88.7\% led to proposal for a regulatory action, in addition to routine pharmacovigilance.

- Safety signals for centrally authorised products result less often in PI updates than safety signals for nationally authorised products.

While previous studies thus showed that the characteristics of drug safety signals affect regulatory actions taken, there is little information on how characteristics of safety signals derived specifically from national SRSs are associated with regulatory actions taken. Actions taken in response to these signals might be dependent on the signal management process which differs for CAPs and NAPs. For this study we therefore focus on signals from the Dutch national SRS and investigate a complete cohort of signals disseminated by Lareb over a period of 10 years. The aim of the study is to characterise signals based on the Dutch SRS and to investigate the association between signal characteristics and PI update stratified by approval type, being centrally authorised products (CAPs) versus nationally and decentralised authorised products (NAPs). As a secondary aim we determine the regulatory actions for signals from the Dutch SRS, including timelines for PI updates.

\section{2 | METHOD}

\subsection{Setting}

For the detection of safety signals, the Netherlands Pharmacovigilance Centre Lareb has historically relied on a case-by-case clinical review of incoming reports, directly submitted by health care professionals (HCP) and consumers. ${ }^{8,9}$ This review is performed by trained pharmacovigilance assessors, the majority of them being medical doctors and pharmacists. Reports that may represent a potential signal in the view of the assessor are discussed in a weekly scientific meeting. Potential signals also undergo a more detailed analysis where signal strength and health impact of drug-AE association are reviewed. Aspects such as clinical quality of the cases, possible confounders, 
clinical pharmacology, disproportionality, information from the literature and other (pharmacovigilance)databases are taken into account. ${ }^{10}$ In addition, a computer-assisted database screening tool is in place as an additional approach to reduce the risk for missing potential signals. This screening tool is a prediction model-based approach to generate a priority list of drug-ADR associations to be analysed. ${ }^{8}$ Signals from the Dutch SRS comprise of both registered and non-registered products such as pharmacy-compounded drugs and herbals and Lareb works in close collaboration with various governmental agencies overseeing these different products. Several factors play a role in determining whether or not a particular signal is worth dissemination to these agencies; The strength of the signal, the unexpected aspect of the ADR, the seriousness of the reaction, and the possibility to implement preventive measures. ${ }^{11}$ In addition, patient perspective plays an important role in the signals that Lareb disseminates. ${ }^{9,12}$ Signals on drugs and vaccines with a marketing authorization are sent to the MEB after these aspects have been discussed in a joined signal detection meeting.

\section{2 | Signals}

This study evaluates all the signals disseminated from the Dutch SRS to the Dutch MEB in the period 2008-2017. A safety signal in our study is defined as information that arises from one or multiple sources (including observations and experiments), which suggests a new potentially causal association, or a new aspect of a known association, between an intervention and an event or set of related events, either adverse or beneficial, that is judged to be of sufficient likelihood to justify verificatory action'. ${ }^{13}$ Signals were retrieved from Lareb's internal analysis and the signal data tracking tool.

Each retrieved signal was characterised on a number of aspects and the signal management process from signal generation to a potential PI update was analysed in four steps:

1. Signal characterisation.

2. Proposed regulatory actions by the Dutch NCA for the signals.

3. Association between signal characteristics and PI update (yes/no).

4. Timing from the moment the signal was issued to $\mathrm{PI}$ update for CAPs.

For step 1-3 we stratify signals in those deriving from CAPs and those deriving from NAPs.

\subsection{1 | Signal characteristics}

Characteristics of the signal were retrieved from Rolfes et al. ${ }^{7}$ and Insani et al. ${ }^{4}$ and included key aspects of the drug used, the experienced $A E$, and strength of evidence for the signal.

Drug characteristics were the ATC-code (first level), ${ }^{14}$ time the drug had been authorised on the Dutch (for NAPs) or European (for CAPs) market in years, the drug being a small molecule drug or a biological using the EMA definitions of a biological 'a medicine whose active substance is made by a living organism, 15 and type of marketing authorization (CAP or NAP).

$A E$ characteristics were the MedDRA system organ class (SOC), ${ }^{16}$ the $A E$ being on the important medical events list (IME) of the EMA, ${ }^{17}$ the AE being a class-effect on ATC-5 level. ${ }^{14}$ For class-effect the same definition used by Insani et al. ${ }^{4}$ was used: 'Knowledge that drugs from the same pharmacological class produce the same adverse effect'. The signals were classified as reflecting possible class effects if the Signal document mentioned that the suspected event is labelled for other drugs from the same class.

Characteristics of the strength of evidence were the number of reports in the signal, the presence of positive dechallenge and/or rechallenge in the reports, mechanistic plausibility of the described association and the average Naranjo score ${ }^{18}$ of the reports in the signal. The Naranjo scores of cases reflect the likelihood of whether an adverse drug reaction is actually due to the drug rather than the result of other factors. A score $\geq 9$ reflects a definite ADR, 5-8 a probable ADR, 1-4 a possible ADR and 0 a doubtful ADR.

\subsection{2 | Proposed regulatory actions}

To determine which regulatory action(s) the MEB proposed, the minutes of the meetings of the Dutch NCA were searched and analysed for discussion of the signals until 31 December 2017. These actions are additional to routine pharmacovigilance, which is ongoing for all reported AEs. The PRAC minutes were also searched for recommendations in relation to additional actions for signals from the Dutch SRS. The PRAC minutes were searched up until April 2018. Classification of the regulatory actions was based on an earlier study looking at signals disseminated from the Dutch SRS. ${ }^{7}$

\subsection{3 | Association characteristics and PI update}

CAPs have a marketing authorization throughout the $\mathrm{EU}^{19}$ and all versions of the PI publicly available on the EMA website. All versions of the PI for CAPs were searched from the moment the signal was sent to the Dutch NCA up until 30th of January 2018 to determine whether the AE in the signal was also present in section 4.8 (Undesirable effects) and/or section 4.4 (Special warning and precautions for use) of the PI.

For NAPs only the latest version of the PI is available on the Dutch NCA website. The latest version of the PI by December 2019 was therefore searched to determine whether the $A E$ in the signal was present in section 4.8 (Undesirable effects) and/or section 4.4 (Special warning and precautions for use).

In case of multiple PIs for a NAP or CAP, because of different manufacturers and/or different administration routes, all the different PIs of the products were searched for changes and a change was recorded if it was made in at least one PI.

We compared the characteristics of signals leading to a PI update, stratified for CAPs and NAPs. 


\section{\begin{tabular}{l|l} 
2.2.4 & Timing of PI change
\end{tabular}}

As $\mathrm{PI}$ versions before and after updates of the $\mathrm{PI}$ are only available for CAPs, we determined the timing from issue of a signal, until the $A E$ was present in the PI for CAPs only. The date of change was the date of procedure finalisation in which the change had been identified. If multiple PIs were updated, the earliest date of update was used to calculate the days it took to change the PI. For the vaccine signals, the brand name of the vaccines used in that period were specified in the signal reports, only the Pls of these brands were checked.

\subsection{Statistics}

For continuous variables, such as time since marketing authorization, a T-test or a nonparametric Mann-Whitney-U test was used depending on the outcome of Levene's test for equality of variances. For other characteristics a Chi-square test was performed or, if the numbers were too small, the Fischer Exact test was used. Overall significance was based on $p<0.05$.

\section{3 | RESULTS}

In the period from 2008 until 2017 a total of 203 signals were disseminated based on the Dutch SRS.

\section{1 | Characteristics}

The most reported ATC class code for drugs was N 'Nervous system' (N) in $24.5 \%$ of the reports. It was followed by Antiinfectives for systemic use (class J), Alimentary tract and metabolism (class A) and Antineoplastic and immunomodulating agents (class $\mathrm{L}$ ), which were reported in $12.0 \%, 10.7 \%$ and $9.4 \%$, respectively. The most frequently reported AEs in the signals were related to the Skin and subcutaneous tissue disorders SOC (26 signals, 12.8\%), followed by the General disorders SOC,
Psychiatric disorders SOC and the Gastrointestinal SOC, reported 24 (11.8\%) and 21 (10.3\%) and 19 (9.4\%) times respectively.

The overall characteristics of the signals, stratified for CAPs versus NAPs, are shown in Table 1. One hundred ninety (93.6\%) of the signals concerned a small molecule drug and 13 (6.4\%) signals a biological, with CAP signals having a lower risk of being a small molecule drug than NAP signals ( $R R=0.81,95 \% \mathrm{Cl} 0.71-0.95)$. For 136 signals (73.5\%) a possible class effect was suggested in the signal. This was more often the case for CAPs than for NAPs ( $R R=1.27,95 \% \mathrm{Cl} 1.06-1.53$ ). In $28 \%$ of the signals a term was listed on the Important Medical Events (IME) list. This was more often the case for signals on CAPs versus NAPs $(R R=1.69,95 \% \mathrm{Cl}$ 1.10-2.61). The majority of signals, 155 (76.3\%) included at least one report with a positive dechallenge. Such positive dechallenges were less common among CAP signals compared to NAP signals ( $R R=0.76,95 \% \mathrm{Cl}$ 0.61-0.93). We did not found a difference between CAPs versus NAPs for positive rechallenges ( $R R=0.86,95 \% \mathrm{Cl} 0.50-1.46$ ) nor for mechanistic plausibility $(\mathrm{RR}=0.94,95 \% \mathrm{Cl} 0.78-1.13)$.

The average number of reports in signals was 15.2 (median 9) with a minimum of 1 and maximum of 327 reports per signal. Twenty-seven signals (13.3\%) consisted of only one or two reports. Most of the signals concerned drugs that had been on the market for 10 years or more (172 signals, $84.7 \%$ ). The median number of years the drugs were on the market was 24.0. Only three drugs were on the market for 1 year or less. CAPs had a shorter period of marketing authorization than NAPs $(p=0.02)$. The average Naranjo score category for reports in the signals was 2 ('possible').

\section{2 | Proposed regulatory actions}

The MEB recommended a total of 256 regulatory actions for the 203 disseminated signals. The maximum number of proposed actions per signal was 3. For three signals from 2009 no action could be determined, because the discussion of the signal was not present in the minutes of the MEB. The most often proposed regulatory action was: 'Signals will be forwarded to LMS/Rapporteur for further evaluations' ( $n=85,32.2 \%)$. This was more often recommended for CAPs than for NAPs $(p=0.003)$. This was followed by 'the MAH is

TABLE 1 Overview of signal characteristics

\begin{tabular}{|c|c|c|c|c|c|}
\hline Characteristic & Total $n=203(\%)$ & CAP $n=61(\%)$ & NAP $n=142(\%)$ & $\begin{array}{l}\text { Risk ratio } \\
\text { (CAP vs. NAP) }\end{array}$ & $95 \% \mathrm{Cl}$ \\
\hline Suspect drug being a small molecule drug & $190(93.6 \%)$ & $49(80.3 \%)$ & $141(99.3 \%)$ & 0.81 & $0.71-0.92$ \\
\hline$A E$ is a class effect & $136(73.5 \%)$ & $48(85.7 \%)$ & $88(68.2 \%)$ & 1.27 & $1.06-1.53$ \\
\hline Mechanistic plausibility & $153(82.7 \%)$ & $44(78.6 \%)$ & $109(84.5 \%)$ & 0.94 & $0.78-1.13$ \\
\hline$A E$ is on the MedDRA IME list & $57(28.1 \%)$ & $24(39.3 \%)$ & $33(23.2 \%)$ & 1.69 & $1.10-2.61$ \\
\hline Signals with a positive Rechallenge & $52(25.6 \%)$ & $14(23.0 \%)$ & $38(26.8 \%)$ & 0.86 & $0.50-1.46$ \\
\hline \multirow[t]{2}{*}{ Signals with a positive Dechallenge } & $155(76.3 \%)$ & $38(62.2 \%)$ & $117(82.3 \%)$ & 0.76 & $0.61-0.93$ \\
\hline & & & & $p$-Value & \\
\hline Median time since marketing authorization (in years) & 24.0 & 16.0 & 25.0 & 0.02 & \\
\hline Mean number of reports per signal & 15.2 & 20.5 & 13 & 0.24 & \\
\hline Average Naranjo score of reports in the signal & 2.0 & 2.1 & 2.0 & 0.21 & \\
\hline
\end{tabular}

Abbreviation: CAP, centrally authorised product. 
requested to include the $\mathrm{AE}$ in the $\mathrm{Pl}$ ' which was recommended $46(18.0 \%)$ times. It should be noted that such a recommendation is only possible if the product has purely national registration in NL without involvement of other countries in Europe. The proposed actions are shown in Table 2, separate for CAPs and NAP signals.

For the 142 NAP signals, a total of 179 regulatory actions were proposed by the MEB. Focusing specifically on PI updates the MEB advised on a PI update for 37 signals. Of these recommendations, 24 signals (64.9\%) resulted in a PI update within the study's timeframe. In total, 87 out of the 142 signals (61.3\%) resulted in a PI update at the time of this study, for instance following the advice of MEB to evaluate the signal in the next PSUR review. Signals from the Dutch SRS can also be further evaluated by the European Medicines Agency Pharmacovigilance Risk Assessment Committee (PRAC) after they have been discussed by the Dutch MEB and are forwarded to the rapporteur. For 23 of the signals disseminated to the MEB, the outcome of a signal discussion could be found in PRAC minutes; 10 of these signals were about centrally authorised products. Figure 1 shows a flowchart of the signals with information on the number of signals (both CAPs and non-CAPs).

\subsection{Association between signal characteristics and PI update}

For 16 out of 61 signals $(26.2 \%)$ concerning CAPs the PI had been updated for either section 4.8 (Adverse drug reactions) or section 4.4 (Special warning and precautions for use). For the 142 NAP signals a total of $87(61.3 \%)$ resulted in PI update at the time of this study.

TAB LE 2 Recommended actions of the MEB for the signals separated for signals of CAPs and NAPs

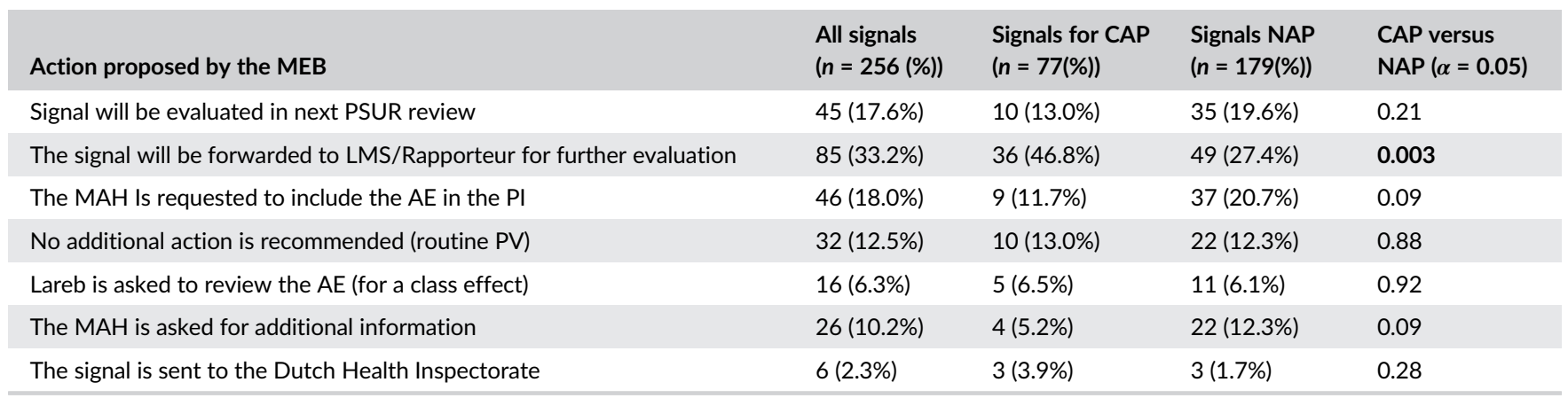

Abbreviation: CAP, centrally authorised product.

$P=0.003$, Significance $P<0.05$.

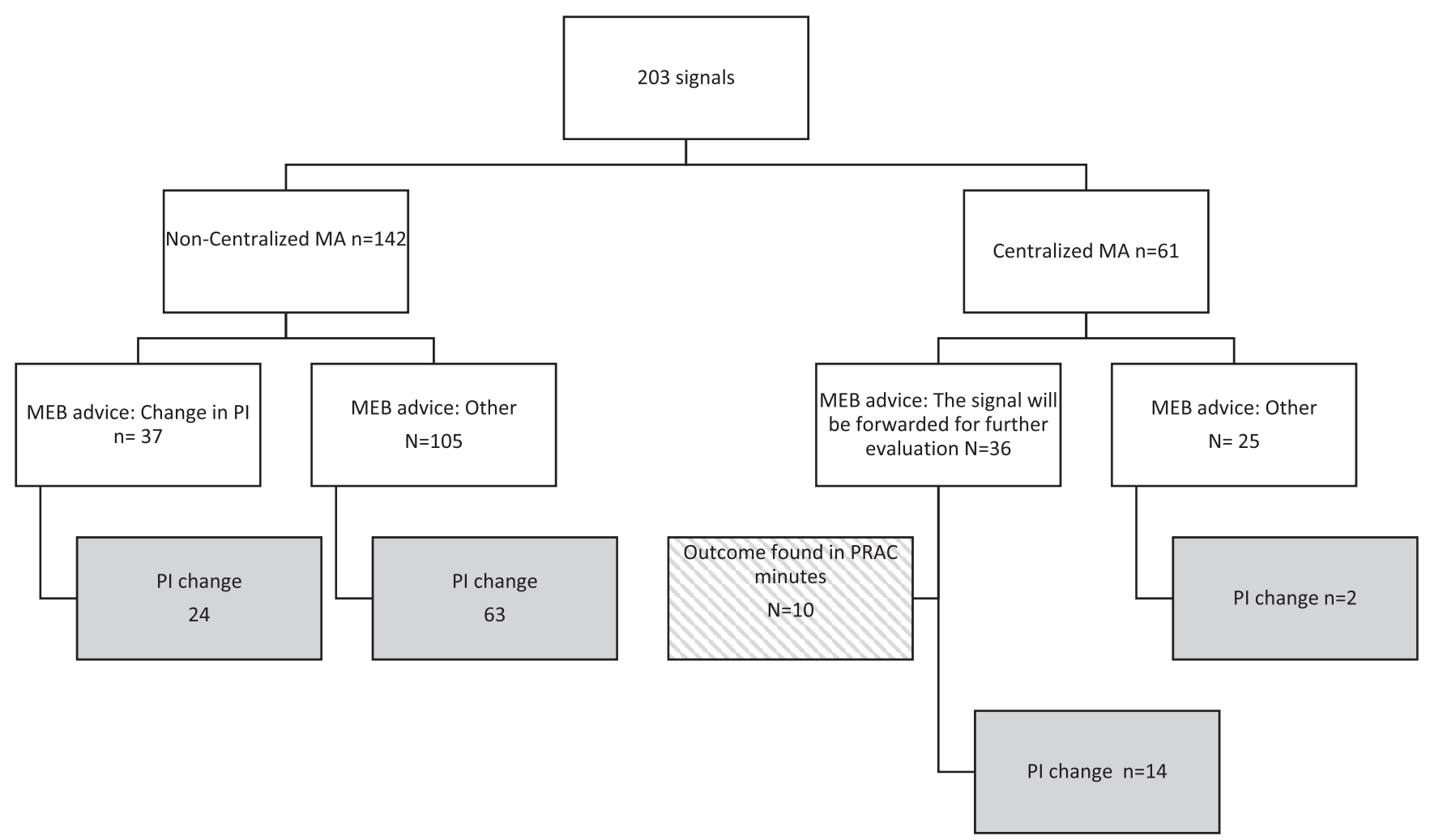

FIGURE 1 Flowchart of the signals 
TAB LE 3 Differences in characteristics for signals with an PI update, stratified for CAPs and non-CAPs

\begin{tabular}{|c|c|c|}
\hline Characteristic & CAP: PI change versus no change & NAP: PI change versus no change \\
\hline AE is a class effect & $100 \%$ versus $67.6 \%, p=0.07^{a}$ & $62.0 \%$ versus $63.6, p=0.99$ \\
\hline Mechanistic plausibility & $81.3 \%$ versus $77.4 \%, p=0.54^{a}$ & $80.4 \%$ versus $74.5 \%, p=0.320$ \\
\hline \% Signals with a positive Rechallenge & $31.3 \%$ versus $41.0 \%, p=0.43^{a}$ & $21.8 \%$ versus $34.5 \%, P=0.046$ \\
\hline \% Signals with a positive Dechallenge & $75 \%$ versus $57.8 \%, p=0.59^{a}$ & $88.6 \%$ versus $72.7 \%, p=0.17$ \\
\hline $\begin{array}{l}\text { Mean time since marketing authorization } \\
\text { (in years) }\end{array}$ & 17.8 versus $22.0, p=0.80^{\mathrm{b}}$ & 28.4 versus $24.6, p=0.21^{\mathrm{b}}$ \\
\hline $\begin{array}{l}\text { Average Naranjo score of reports in the } \\
\text { signal }\end{array}$ & 1.9 versus $2.2, p=0.59^{\mathrm{b}}$ & 2.0 versus $2.0, p=0.73^{b}$ \\
\hline
\end{tabular}

Note: Results in bold are significant $(\alpha=0.05)$.

${ }^{a}$ Fischer exact test.

${ }^{\mathrm{b}}$ Non-parametric Mann-Whitney-U test.

TAB LE 4 Signals concerning centrally authorised products with change in PI

\begin{tabular}{|c|c|c|c|c|}
\hline Signal & Date of signal & Date of change & Days to change & $\begin{array}{l}\text { Section of } \\
\text { change }\end{array}$ \\
\hline 1. Adalimumab and neuroendocrine carcinoma of the skin & 10 February 2008 & 26 November 2012 & 1751 & 4.4 and 4.8 \\
\hline 3. Loratadine, desloratadine and convulsions ${ }^{a}$ & 16 July 2008 & $\begin{array}{r}25 \text { February } 2012 \\
(23 \text { May 2017) }\end{array}$ & $1319(3233)^{\mathrm{a}}$ & 4.4 and 4.8 \\
\hline $\begin{array}{l}\text { 4. Antiepileptic drugs and increased risk of suicidal } \\
\text { behaviour and suicidal ideation }\end{array}$ & 3 September 2008 & 4 February 2009 & 154 & 4.4 \\
\hline 5. Antipsychotic drugs and hypothermia & 3 September 2008 & 2 July 2012 & 1398 & 4.8 \\
\hline 6. Angiotensin-II receptor antagonists and paraesthesia & 21 October 2008 & 25 November 2008 & 35 & 4.8 \\
\hline 7. Adalimumab and pustular psoriasis & 6 July 2009 & 1 September 2009 & 57 & 4.8 \\
\hline 8. Pantoprazole and oral adverse drug reactions & 9 February 2010 & 7 January 2011 & 332 & 4.8 \\
\hline 12. Rivastigmine and nightmares and abnormal dreams & 22 May 2014 & 23 November 2015 & 550 & 4.8 \\
\hline 13. Fluticasone and palpitations & 16 October 2014 & 10 November 2015 & 390 & 4.8 \\
\hline $\begin{array}{l}\text { 14. Dimethyl fumarate and progressive multifocal } \\
\text { leukoencephalopathy (PML) }\end{array}$ & 18 February 2015 & 22 December 2015 & 307 & 4.4 and 4.8 \\
\hline 15. Proton pump inhibitors and fundic gland polyps & 8 September 2015 & 03 May 2017 & 603 & 4.8 \\
\hline 16. Desloratadine and abnormal behaviour-update cases & 19 September 2016 & 23 May 2017 & 246 & 4.8 \\
\hline
\end{tabular}

abetween brackets the date of change of section 4.4 .

Table 3 shows that no significant differences in characteristics were seen for both CAPs and NAPs between signals leading to an PI update and those that did not.

\subsection{Timing of PI change}

For CAPs the time to first PI update after the signal was sent to the MEB was a median of 361 days (minimum 35-maximum 1751 days). In Table 4 the signals which led to a change in PI are shown including the date the signal was issued and the date of change. For NAPs timing to PI update could not be determined.

\section{4 | DISCUSSION}

NCAs play an important role in detecting safety signals. A previous study found that the United Kingdom, Germany, France, The Netherlands, Spain, Denmark, Portugal, and Italy are notable contributors of safety signals in the European pharmacovigilance system. ${ }^{20}$ The current study characterised the signals from the Dutch SRS, including proposed actions by the Dutch NCA following a signal and whether signals resulted in product information updates for both centrally authorised products (CAPs) and nationally and decentrally authorised products (NAPs). 
Our study found that almost $70 \%$ of the signals from the Dutch SRS concern signals for NAPs. Most of the AEs in these signals (71.9\%) were not included on the Important Medical Events (IME) list ${ }^{17}$ and signals for CAPs more often concerned AES on the IME list than signals for NAPs. However, these AEs may be perceived as burdensome and severe by patients, even though they might not be serious from a medical or regulatory point of view. ${ }^{21}$ We also found other differences in the characteristics of signals for CAPs vs. NAPs; CAPs were less often small molecule drugs than non-CAPs and more class-effects were described in the signals of CAPs. This is not surprising as entire newer classes of biological drugs have come on the market which require authorization via the centralised procedure. Time since marketing authorization was also significantly shorter for signals on CAPs than non-CAPs, which is not surprising since CAPS are often newer products. For signals on these newer products, Lareb often focuses on mechanistic plausibility or possible class-effects. The number of signals with positive dechallenges is higher for non-CAPs. Information on positive rechallenges is far less often available, but more so for CAPs than for non-CAPs. It could be that for CAPs, because they are newer products, a rechallenge is more often performed.

Overall, when looking at the most reported organ classes in the signals from the Dutch SRS, the results are similar to those evaluated by the PRAC during 2012-2016. ${ }^{4}$ Signals from the Dutch SRS are less often on drugs in the class Antineoplastic and immunomodulating agents, which often contains biologicals, then those evaluated by the PRAC. ${ }^{4}$ However, the focus of the Dutch pharmacovigilance centre has recently shifted more towards gaining knowledge on these products through the use of registries and an intensive monitoring study on drugs used in immune-mediated inflammatory disease. ${ }^{22-24}$

Signals found through the Dutch SRS can lead to multiple regulatory actions. The Dutch NCA MEB plays a pivotal role in this process, as they decide which action is suitable for the different signals. ${ }^{7}$ However, for products without pure national registration in NL, the recommendations of the MEB are not definitive, as the signals need to be further discussed at European level and are often assessed by different countries. Lareb signals resulted in PI update for $26 \%$ of signals concerning CAPs and in $61.3 \%$ non-CAPs. One of the reasons for this difference could be that signals for NAPs include discrepancies between European PI texts; for instance when the $A E$ is already labelled in other European countries but not yet in the Dutch PI. MAHs can then be asked to align the Dutch PI with PIs from surrounding countries. For NAPs the regulatory decision proposed by the MEB was to include the AE in the $\mathrm{PI}$ in $20.7 \%$ of signals. Other proposed regulatory actions such as requests for additional information and forwarding of the signal to lead member states could ultimately also lead to a PI update.

Previously it was found that drug safety signals characteristics that were predictors for PI update at PRAC level were the presence of evidence in multiple types of data sources, mechanistic plausibility of the signalled association, seriousness of the event, and age of the drug. ${ }^{4}$ However, in this study the characteristics in Table 3 were similar for signals for which the PI was updated and for those were this was not the case. The assessment of signals often combines different forms of evidence and individual characteristics of the SRS signal may therefore not be strong predictors. Another factor could be the selection mechanism of signals at Lareb, using a Signal Impact Assessment Tool (SIAT) as aid in signal selection, which prioritises signals based on signal strength and health impact. ${ }^{10}$ This could explain that the signals selected from the SRS are relatively similar for the CAPs and NAPs, regardless of the PI update. It should be noted that the signal from the SRS is not necessary the sole contributor to a PI update. Most often following a Lareb signal, additional data is requested from the MAH and only after evaluation of the additional data a recommendation is made.

For the CAPs we were able to investigate the timing between issue of a signal from Lareb to the MEB and the PI update. There is a large variation in time to $\mathrm{PI}$ update. For some signals this was very short (35 days). This is most probably due to the fact that the PI had been updated independent from a Lareb signal. Also long periods until PI update were seen. For instance a signal on Desloratadine and increased appetite, ${ }^{25}$ which was discussed at PRAC 6 years after the signal of Lareb on this association $^{26}$ because new data became available through other sources than the Dutch SRS. For products with a national marketing authorization, we could only assess if the safety issue from the signal has been included in the $\mathrm{Pl}$ at the moment of the study, but not how long this took.

\section{1 | Strengths and limitations}

The major strength of this study is that it examined all safety signals disseminated from the Dutch SRS over a period of 10 years. However, this study also has some limitations; foremost the dataset, especially for CAPs with a PI change is limited in size. Secondly, we used publicly available data, therefore not all information was available, for example, the discussion of Lareb signals during the PSUR procedure cannot be found in the publicly available PRAC minutes unless the signal led to a product information update. Insani et al. ${ }^{4}$ used 'Presence of disproportionate reporting' as a characteristic for the strength of evidence in their study on signal characteristics associated with PI updates following signal evaluation by the European Medicines Agency Pharmacovigilance Risk Assessment Committee. However for the Dutch Signals it was difficult to retrospectively obtain information on the measure of disproportionality for the full cohort of Signals. Therefor this characteristic was not included. Lastly, the time to change of the PI was often long and it could not be determined that this change was caused by the signal of Lareb or by other sources, such as signals from other pharmacovigilance centres or by literature research or a combination of these.

Signal detection and subsequent regulatory actions are core activities in pharmacovigilance which make it possible to rapidly deliver new information on the safety of medicines from real-world data which enables us to fill knowledge gaps. ${ }^{27}$ Ultimately, pharmacovigilance activities and regulatory actions aim to reduce harm by improving the use of medicines and patient care. By measuring the harm reduced by these regulatory activities, we generate evidence about the effectiveness and show the effect of these activities. ${ }^{28,29}$ From our current study it is not possible to estimate the effects on patient care. 


\section{5 | CONCLUSION}

The Dutch Spontaneous Reporting System and National Competent Authority have an important role in detecting signals, especially for products without a centralised marketing authorization. Almost $70 \%$ of the signals from the Dutch SRS concern products without a centralised marketing authorization. $88.7 \%$ of the signals from the Dutch SRS in the period from 2008 to 2017 led to a recommendation for a regulatory action by the MEB in addition to routine pharmacovigilance.

There are some notable differences in characteristics of the disseminated safety signals between CAPs and NAPs. Signals for NAPs more often led to a PI update than signals for CAPs.

\section{CONFLICT OF INTEREST}

The authors declare no conflict of interest.

\section{ETHICS STATEMENT}

The authors state that no ethical approval was needed.

\section{ORCID}

Florence van Hunsel (iD) https://orcid.org/0000-0001-8965-3224

\section{REFERENCES}

1. Lester J, Neyarapally GA, Lipowski E, Graham CF, Hall M, Dal PG. Evaluation of FDA safety-related drug label changes in 2010. Pharmacoepidemiol Drug Saf. 2013;22(3):302-305.

2. Pacurariu AC, Coloma PM, van HA GG, Sturkenboom MC, Straus SM. A description of signals during the first 18 months of the EMA pharmacovigilance risk assessment committee. Drug Saf. 2014;37(12): 1059-1066.

3. Raine J. Risk management: a European Regulatory view. In: Mann RM, Andrews EB, eds. Pharmacovigilance. 2nd ed. New Jersey, USA: John Wily \& Sons Ltd.; 2007:553-558.

4. Insani WN, Pacurariu AC, Mantel-Teeuwisse AK, GrossMartirosyan L. Characteristics of drugs safety signals that predict safety related product information update. Pharmacoepidemiol Drug Saf. 2018;27(7):789-796

5. European Medicines Agency (EMA). Safety signal 2021; 2021. https://www.ema.europa.eu/en/glossary/safety-signal

6. Santoro A, Genov G, Spooner A, Raine J, Arlett P. Promoting and protecting public health: how the European Union Pharmacovigilance System Works. Drug Saf. 2017;40(10):855-869.

7. Rolfes L, Kolfschoten J, van Hunsel F, Kooijman M, van Puijenbroek E. Actions in response to drug safety signals arising from a spontaneous reporting system: retrospective study in The Netherlands. Int J Risk Saf Med. 2016;28(2):115-123.

8. Scholl JHG, van Hunsel F, Hak E, van Puijenbroek EP. A prediction model-based algorithm for computer-assisted database screening of adverse drug reactions in The Netherlands. Pharmacoepidemiol Drug Saf. 2018;27(2):199-205

9. van Hunsel F, de Waal S, Harmark L. The contribution of direct patient reported ADRs to drug safety signals in The Netherlands from 2010 to 2015. Pharmacoepidemiol Drug Saf. 2017;26(8):977-983.

10. Rolfes L, Kolfschoten J, van Hunsel F, van Puienbroek E. The validity and reliability of a signal impact assessment tool. Pharmacoepidemiol Drug Saf. 2016;25(7):815-819

11. Graham DJ, Waller PC, Kurz X. A View From Regulatory Agencies. In Pharmacoepidemiology, B.L. Strom (Ed.). Pharmacoepidemiology. 2000;109-124. https://doi.org/10.1002/0470842555.ch8
12. van Hunsel F, Harmark L, Rolfes L. Fifteen years of patient reporting -what have we learned and where are we heading to? Expert Opin Drug Saf. 2019;18(6):477-484.

13. CIOMS Working Group VIII. Practical Aspects of Signal Detection in Pharmacovigilance: Report of CIOMS Working Group VIII. Geneva. Report No.: 9290360828; 2010.

14. WHO Collaborating Centre for Drug Statistics Methodology. The ATC/DDD system; 2008. http://wwwwhoccno/atcddd/

15. European Medicines Agency (EMA). Biological medicine 2020; 2020. https://www.ema.europa.eu/en/glossary/biological-medicine

16. Brown EG, Wood L, Wood S. The medical dictionary for regulatory activities (MedDRA). Drug Saf. 1999;20(2):109-117.

17. European Medicines Agency (EMA). Important medical event terms list (MedDRA version 19.1); 2016. www.ema.europa.eu/docs/en_ GB/document_library/Other/2016//WC500208836xls

18. Naranjo CA, Busto U, Sellers EM, et al. A method for estimating the probability of adverse drug reactions 1. Clin Pharmacol Ther. 1981;30 (2):239-245.

19. Zeitoun JD, Lefevre JH, Downing NS, Bergeron H, Ross JS. Regulatory review time and post-market safety events for novel medicines approved by the EMA between 2001 and 2010: a cross-sectional study. Br J Clin Pharmacol. 2015;80(4):716-726.

20. Farcas A, Mahalean A, Bulik NB, Leucuta D, Mogosan C. New safety signals assessed by the Pharmacovigilance Risk Assessment Committee at EU level in 2014-2017. Expert Rev Clin Pharmacol. 2018;11(10):10451051

21. Frankenfeld C. 'serious' and 'severe' adverse drug reactions need defining. BMJ. 2004;329(7465):573.

22. van Lint JA, Jessurun NT, Hebing RCF, et al. Patient-reported burden of adverse drug reactions attributed to biologics used for immunemediated inflammatory diseases. Drug Saf. 2020;43(9):917-925.

23. Giraud EL, Jessurun NT, van Hunsel F, et al. Frequency of real-world reported adverse drug reactions in rheumatoid arthritis patients. Expert Opin Drug Saf. 2020;19(12):1617-1624.

24. Giraud EL, Thomas PWA, van Lint JA, et al. Adverse drug reactions from real-world data in inflammatory bowel disease patients in the IBDREAM registry. Drug Saf. 2021. https://doi.org/10.1007/s40264021-01045-3

25. Netherlands Pharmacovigilance Centre Lareb. Desloratadine and increased appetite [Signal]. 2011

26. European Medicines Agency (EMA). PRAC recommendations on signals-Adopted at the 25-29 September 2017 PRAC meeting 2017; 2017. https://databankws.lareb.nl/Downloads/kwb_2011_3 deslo.pdf. accessed October 92020

27. Potts J, Genov G, Segec A, Raine J, Straus S, Arlett P. Improving the safety of medicines in the European Union: from signals to action. Clin Pharmacol Ther. 2020;107(3):521-529.

28. van Hunsel F, Gardarsdottir H, de Boer A, Kant A. Measuring the impact of pharmacovigilance activities, challenging but important. $\mathrm{Br} J$ Clin Pharmacol. 2019;85(10):2235-2237.

29. van Hunsel F, Peters L, Gardarsdottir H, Kant A. Assessing the impact on health of pharmacovigilance activities: example of four safety signals. Drug Saf. 2021. https://doi.org/10.1007/s40264-021-01047-1.

How to cite this article: van Hunsel F, de Jong E, GrossMartirosyan L, Hoekman J. Signals from the Dutch national spontaneous reporting system: Characteristics and regulatory actions. Pharmacoepidemiol Drug Saf. 2021;30:1115-1122. https://doi.org/10.1002/pds.5246 